\title{
Location and stability of the triangular Lagrange points in photo-gravitational elliptic restricted three body problem with the more massive primary as an oblate spheroid
}

\author{
A. Arantza Jency ${ }^{1}$, Ram Krishan Sharma ${ }^{1}$ * \\ ${ }^{1}$ Department of Aerospace Engineering, Karunya Institute of Technology and Sciences, Coimbatore-641114, Tamil Nadu, India \\ *Corresponding author E-mail: ramkrishansharma@gmail.com
}

\begin{abstract}
The triangular Lagrangian points of the elliptic restricted three-body problem (ERTBP) with oblate and radiating more massive primary are studied. The mean motion equation used here is different from the ones employed in many studies on the perturbed ERTBP. The effect of oblateness on the mean motion equation varies. This change influences the location and stability of the triangular Lagrangian points. The points tend to shift in the y-direction. The influence of the oblateness on the critical mass ratio is also altered. But the eccentricity limit for stability remains the same.
\end{abstract}

Keywords: Mean Motion; Planar ERTBP; Oblateness; Radiation Pressure; Critical Mass Ratio; Triangular Lagrangian Points; Transition Curves.

\section{Introduction}

Because of their stable location (due to nullified centrifugal and gravitational forces [1]) and many other advantages particular to the chosen system and the positions, the Lagrangian points are of practical interests from the late 20th century [2]. They serve to be the address for many natural (Trojan asteroids) and artificial satellites. Space agencies across the world try to utilize these points to the fullest. Moreover, the triangular Lagrangian points get added attention as they tend to be more stable. The OSIRIS-REX mission of NASA and Hayabusa2 of the Japanese Space Agency manifest the interest for triangular Lagrangian points.

The history of Lagrangian points dates back to the $18^{\text {th }}$ century when Euler and Lagrange discovered the five Lagrangian points. In the early studies, the theory considered the orbit of the primaries circular [3, 4]. Later investigations included the eccentricity effects on the Lagrangian points [5], [6]. The research was still very general that any two systems with the same eccentricity led to the same Lagrangian points. Then, the points became system-specific with the inclusion of perturbations [7 - 10]. These perturbations directly affected the equations of motion and the mean motion equation, which in turn influenced the properties of the Lagrangian points.

A recent study [11] recognised and rectified the defects of the mean motion equation used in earlier investigations. This paper intends to employ the newly formulated mean motion equation [11] to the elliptic restricted three-body problem (ERTBP) considering the more massive primary as an oblate and radiating spheroid and the other primary as a point mass. The changes imposed on the location and stability of the triangular Lagrangian points are studied.

Firstly, the equations of motion and the mean motion equation for the particular case are formulated. The triangular Lagrangian points are located. The critical mass ratio and the limiting eccentricity for the system to be stable are determined. Finally, the transition curves are generated to collectively show the region of stability in the eccentricity - mass ratio plane.

\section{Equations of motion}

Consider an object with a negligible mass in the plane of two other bodies called the primaries (masses $\mu$ and $1-\mu$ ). The gravitational forces of the primaries affect the motion of the third body (with negligible mass) but, the third body does not affect the primaries. The origin of this system is at the barycentre. The $\mathrm{x}$-axis joins the primaries. The line running perpendicular to it forms the $y$-axis. Let the more massive primary be an oblate (oblateness coefficient $\mathrm{A}_{1}$ ) radiating (radiation pressure $\mathrm{q}$ ) spheroid and the other one a point mass. The primaries revolve around their barycentre in an elliptic orbit (eccentricity e and true anomaly $\theta$ ). Let the third body be at distances $r_{1}$ and $\mathrm{r}_{2}$ from the primaries which are given by

$$
\begin{aligned}
& r_{1}^{2}=(x-\mu)^{2}+y^{2} \\
& r_{2}^{2}=(x+1-\mu)^{2}+y^{2}
\end{aligned}
$$


The equation of the third body can be represented in the pulsating dimensionless coordinate system [3] as

$\begin{aligned} \mathrm{x}^{\prime \prime}-2 \mathrm{y}^{\prime} & =\frac{1}{(1+\mathrm{e} \cos \theta)} \Omega_{\mathrm{x}} \\ \mathrm{y}^{\prime \prime}+2 \mathrm{x}^{\prime} & =\frac{1}{(1+\mathrm{e} \cos \theta)} \Omega_{\mathrm{y}} .\end{aligned}$

where $\Omega$ is the force function given by

$\Omega=\frac{1}{2}\left(\mathrm{x}^{2}+\mathrm{y}^{2}\right)+\frac{1}{\mathrm{n}^{2}}\left(\frac{(1-\mu) \mathrm{q}}{\mathrm{r}_{1}}+\frac{\mu}{\mathrm{r}_{2}}+\frac{(1-\mu) \mathrm{qA} \mathrm{A}_{1}}{2 \mathrm{r}_{1}{ }^{3}}\right)$.

\section{Mean motion}

The mean motion equations used in the perturbed ERTBP face numerous defects [11]. The rectified mean motion equation is used here. This mean motion equation is obtained from the secular perturbation effects of oblateness [12].

$\mathrm{n}^{2}=1+2 \mathrm{~K}_{1} \mathrm{~A}_{1}\left(1+\frac{7}{4} \mathrm{e}^{2}\right)$

where $K_{1}$ is a combination of the equatorial radius of the more massive primary $\left(R_{e}\right)$, the distance between the primaries $(R)$ and the semimajor axis (a).

$\mathrm{K}_{1}=\frac{3 \mathrm{R}^{2}}{\mathrm{a}^{2} \mathrm{R}_{\mathrm{e}}^{2}}$

\section{Location of triangular points}

The Lagrangian points are the singularity points where the derivatives of the force function become zero.

$\Omega_{\mathrm{x}}=0$,

$x-\frac{1}{n^{2}}\left[\frac{(1-\mu) q(x-\mu)}{r_{1}{ }^{3}}+\frac{\mu(x+1-\mu)}{r_{2}{ }^{3}}+\frac{3 A_{1}}{2} \frac{(1-\mu) q(x-\mu)}{r_{1}{ }^{5}}\right]=0$.

And

$\Omega_{\mathrm{y}}=0$,

$\mathrm{y}\left[1-\frac{1}{\mathrm{n}^{2}}\left(\frac{(1-\mu) \mathrm{q}}{\mathrm{r}_{1}{ }^{3}}+\frac{\mu}{\mathrm{r}_{2}{ }^{3}}+\frac{3}{2} \mathrm{~A}_{1} \frac{(1-\mu) \mathrm{q}}{\mathrm{r}_{1}{ }^{5}}\right)\right]=0$.

For the triangular Lagrangian points $y \neq 0$ because, they do not lie on the x-axis. Instead, they form nearly equilateral triangles [7] with the primaries. Therefore, the above equations take the form

$x-\frac{1}{n^{2}}\left[\frac{(1-\mu) q(x-\mu)}{r_{1}{ }^{3}}+\frac{\mu(x+1-\mu)}{r_{2}{ }^{3}}+\frac{3 A_{1}}{2} \frac{(1-\mu) q(x-\mu)}{r_{1}{ }^{5}}\right]=0$,

$\left[1-\frac{1}{\mathrm{n}^{2}}\left(\frac{(1-\mu) \mathrm{q}}{\mathrm{r}_{1}{ }^{3}}+\frac{\mu}{\mathrm{r}_{2}{ }^{3}}+\frac{3}{2} \mathrm{~A}_{1} \frac{(1-\mu) \mathrm{q}}{\mathrm{r}_{1}{ }^{5}}\right)\right]=0$

Eqs. (6) should be solved to get the location of the triangular Lagrangian points. The value of radiation pressure q is nearly 1 . So, in further calculations, to ease the derivation, let us replace it with the term $(1-\epsilon)$. Therefore, $q=1-\epsilon$. Substituting in the above equations

$x-\frac{1}{n^{2}}\left[\frac{(1-\mu)(1-\epsilon)(x-\mu)}{r_{1}{ }^{3}}+\frac{\mu(x+1-\mu)}{r_{2}{ }^{3}}+\frac{3 A_{1}}{2} \frac{(1-\mu)(1-\epsilon)(x-\mu)}{r_{1}{ }^{5}}\right]=0$,

$\left[1-\frac{1}{\mathrm{n}^{2}}\left(\frac{(1-\mu)(1-\epsilon)}{\mathrm{r}_{1}{ }^{3}}+\frac{\mu}{\mathrm{r}_{2}{ }^{3}}+\frac{3}{2} \mathrm{~A}_{1} \frac{(1-\mu)(1-\epsilon)}{\mathrm{r}_{1}{ }^{5}}\right)\right]=0$.

Solving Eqs. (7),

$2 \mathrm{n}^{2} \mathrm{r}_{1}{ }^{5}-2 \mathrm{r}_{1}^{2}(1-\epsilon)-3 \mathrm{~A}_{1}(1-\epsilon)=0$,

$\mathrm{n}^{2} \mathrm{r}_{2}{ }^{3}-1=0$.

Let $r_{1}=1+\delta_{1}$ as it is slightly more than the value 1 . Solving Eq. (8), we get

$\delta_{1}=\frac{2-2 \epsilon+3 \mathrm{~A}_{1}-3 \mathrm{~A}_{1} \epsilon-2 \mathrm{n}^{2}}{5 \mathrm{n}^{2}-2+2 \epsilon}$

$\delta_{1}=\mathrm{A}_{1}-\frac{4}{3} \mathrm{~K}_{1} \mathrm{~A}_{1}\left(1+\frac{7}{4} \mathrm{e}^{2}\right)-\frac{2}{3} \epsilon-\frac{5}{3} \mathrm{~A}_{1} \epsilon+\frac{28}{9} \mathrm{~K}_{1} \mathrm{~A}_{1} \epsilon\left(1+\frac{7}{4} \mathrm{e}^{2}\right)+\frac{4}{9} \epsilon^{2}$, 
$\mathrm{r}_{1}^{2}=1+\mathrm{A}_{1}-\frac{4}{3} \mathrm{~K}_{1} \mathrm{~A}_{1}\left(1+\frac{7}{4} \mathrm{e}^{2}\right)-\frac{2}{3} \epsilon-\frac{5}{3} \mathrm{~A}_{1} \epsilon+\frac{28}{9} \mathrm{~K}_{1} \mathrm{~A}_{1} \epsilon\left(1+\frac{7}{4} \mathrm{e}^{2}\right)+\frac{4}{9} \epsilon^{2}$

Similarly, let $r_{2}=1-\delta_{2}$ because this will be slightly less than 1 . Solving Eq. (9), we get

$$
\begin{aligned}
& \delta_{2}=\frac{4}{3} \mathrm{~K}_{1} \mathrm{~A}_{1}\left(1+\frac{7}{4} \mathrm{e}^{2}\right), \\
& \mathrm{r}_{2}{ }^{2}=1-\frac{4}{3} \mathrm{~K}_{1} \mathrm{~A}_{1}\left(1+\frac{7}{4} \mathrm{e}^{2}\right) .
\end{aligned}
$$

Comparing Eqs. (10) and (11) with Eqs. (1) and (2) and solving for x and y, we get the location of the triangular Lagrangian points.

$\mathrm{x}=\mu-\frac{1}{2}-\frac{\mathrm{A}_{1}}{2}+\frac{1}{3} \epsilon$

$y= \pm \frac{\sqrt{3}}{2}\left[1+\frac{A_{1}}{3}-\frac{8}{9} K_{1} A_{1}\left(1+\frac{7}{4} e^{2}\right)\right]-\frac{2}{9} \epsilon$

There is a shift observed in the y-coordinates of the points when compared to the earlier studies [8], [9]. When these perturbations are removed, the locations match with the classical ERTBP results [3], [4].

\section{Stability of triangular points}

An object is said to be in equilibrium if it stays in the same position for infinite time. As this is not practically possible, it is good if at least these points are stable. To investigate the stability of the points, we analyse the motion of the third body in a place very close (with a very small displacement $(\xi, \eta))$ to the location of the triangular Lagrangian points $(a, b)$. The motion of the third body in the vicinity of the Lagrangian points is defined by the variational differential equations

$\xi^{\prime \prime}-2 \eta^{\prime}=\frac{1}{1+e \cos \theta}\left[\xi \Omega_{x x}+\eta \Omega_{x y}\right]$

$\eta^{\prime \prime}+2 \xi^{\prime}=\frac{1}{1+e \cos \theta}\left[\xi \Omega_{\mathrm{xy}}+\eta \Omega_{\mathrm{yy}}\right]$

Substituting the general solutions of these equations, we can get the characteristic equation representing the motion.

$\lambda^{4}+\left(4-\frac{\Omega_{\mathrm{xx}}}{\sqrt{1-\mathrm{e}^{2}}}-\frac{\Omega_{\mathrm{yy}}}{\sqrt{1-\mathrm{e}^{2}}}\right) \lambda^{2}+\left(\frac{\Omega_{\mathrm{xx}} \Omega_{\mathrm{yy}}-\Omega_{\mathrm{xy}}{ }^{2}}{\left(1-\mathrm{e}^{2}\right)}\right)=0$.

This equation is of the form

$a \lambda^{4}+b \lambda^{2}+c=0$

And its solution

$\lambda= \pm \sqrt{\frac{-\mathrm{b} \pm \sqrt{\mathrm{b}^{2}-4 \mathrm{ac}}}{2 \mathrm{a}}}$

where

$\mathrm{a}=1$,

$\mathrm{b}=4-\frac{\Omega_{\mathrm{xx}}}{\sqrt{1-\mathrm{e}^{2}}}-\frac{\Omega_{\mathrm{yy}}}{\sqrt{1-\mathrm{e}^{2}}}$

$\mathrm{c}=\frac{\Omega_{\mathrm{xx}} \Omega_{\mathrm{yy}}-\Omega_{\mathrm{xy}}{ }^{2}}{\left(1-\mathrm{e}^{2}\right)}$,

and

$\Omega_{\mathrm{xx}}=\frac{3}{4}+\mathrm{A}_{1}\left[\frac{3}{2}-3 \mu+\mathrm{K}_{1}\left(1+\frac{7}{4} \mathrm{e}^{2}\right)\right]+\epsilon\left[-\frac{1}{2}+\frac{3}{2} \mu\right]$,

$\Omega_{\mathrm{yy}}=\frac{9}{4}+\mathrm{A}_{1}\left[\frac{3}{2}-\mathrm{K}_{1}\left(1+\frac{7}{4} \mathrm{e}^{2}\right)\right]+\epsilon\left[\frac{1}{2}-\frac{3}{2} \mu\right]$,

$\Omega_{\mathrm{xy}}= \pm \frac{\sqrt{3}}{2}\left[3 \mu-\frac{3}{2}+\mathrm{A}_{1}\left[\mu-2-\frac{2}{3} \mathrm{~K}_{1}\left(1+\frac{7}{4} \mathrm{e}^{2}\right)+\frac{4}{3} \mu \mathrm{K}_{1}\left(1+\frac{7}{4} \mathrm{e}^{2}\right)\right]+\epsilon\left[\frac{1}{4}+\frac{\mu}{4}\right]\right]$

The roots of the characteristic equation are given by

$\lambda= \pm \sqrt{\frac{-b \pm \sqrt{b^{2}-4 a c}}{2 a}}$ 
It is not possible for this system to be asymptotically stable. To attain marginal stability, all the roots should be imaginary. There are two conditions to be satisfied for the roots to be imaginary.

The first condition is that $b>0$. Considering this, the limiting eccentricity $e_{0}$ can be found. If the eccentricity of the orbit of the primaries lie in the range $0 \leq e \leq e_{0}$, then the system will be stable.

$$
\begin{aligned}
& e^{2}<1-\frac{9}{16}\left[1+A_{1}-\mu A_{1}\right]^{2}, \\
& e_{0}=\sqrt{1-\frac{9}{16}\left[1+A_{1}-\mu A_{1}\right]^{2}} .
\end{aligned}
$$

This is the same equation obtained in [9]. This means that neither the radiation pressure nor the change in the mean motion equation impacts the limiting eccentricity. When the oblateness is zero, the limiting eccentricity gets the value $\sqrt{7} / 4$ (same as [6]). The value decreases with increasing oblateness. But this limiting eccentricity is unaltered by the radiation pressure of the more massive primary.

The second condition needs the discriminant of the system to be greater than zero $\left(b^{2}-4 a c \geq 0\right)$. With this condition, the critical mass ratio obtained here is

$$
\mu_{c}=\mu_{e}+A_{1}\left(-\frac{1}{9}-\frac{11}{9 \sqrt{69}}-\frac{8}{27 \sqrt{69}} K_{1}\right)-\frac{2}{27 \sqrt{69}} \epsilon .
$$

Where, $\mu_{e}$ is the result of unperturbed ERTBP (from [6])

$$
\mu_{e}=\frac{1}{54}\left(27-3 \sqrt{3} \sqrt{-73+64 e^{2}+96 \sqrt{1-e^{2}}}\right) \text {. }
$$

This critical mass ratio varies slightly from that obtained in previous studies [9], [10]. This variation is due to the change in the mean motion.

Fig. 1 shows the variation of $\mu_{c}$ with eccentricity for different values of $\epsilon$ and $A_{1}$. The critical mass ratio decreases with eccentricity, oblateness and also the radiation pressure.

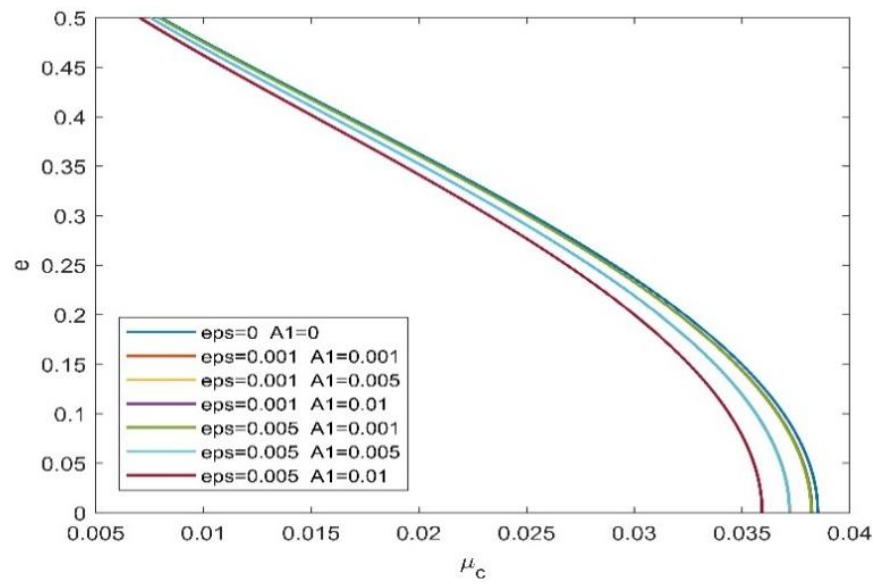

Fig. 1: Variation of Critical Mass Ratio $\left(\mu_{c}\right)$ with Eccentricity (E) for Different $A_{1}$ and $\epsilon$.

\section{Transition curves}

Transition curves show the region within which the triangular Lagrangian points remain stable in the $\mu-e$ plane. The method suggested in [13] is used to get the transition curves. The variational equations of motion are written in the form of a matrix. Its characteristic exponents can be expanded as

$\lambda=\lambda_{0}+\lambda_{1} e+\lambda_{2} e^{2}+\cdots$

Here, the higher order terms are truncated and the solutions for $\lambda_{0}, \lambda_{1}$ and $\lambda_{2}$ are obtained by equating the coefficients of e terms in the general solution of variational equations of motion.

$\lambda_{0}$ is the solution of the equation

$\lambda_{0}{ }^{4}+\left(4-\Omega_{x x}-\Omega_{y y}\right) \lambda_{0}{ }^{2}+\left(\Omega_{x x} \Omega_{y y}-\Omega_{x y}{ }^{2}\right)=0$,

$\lambda_{1}=0$

$\lambda_{2}=-\left[\frac{\left(Q^{2}-4 R-16\right) \lambda_{0}{ }^{2}+A_{0} F_{0}+A_{1} F_{1}+A_{2} F_{2}}{4\left(Q^{2}-4 Q-4 R\right) \lambda_{0}{ }^{2}+32 R}\right] \lambda_{0}$,

where,

$Q=-\left(4-\Omega_{x x}-\Omega_{y y}\right)$,

$R=\Omega_{x x} \Omega_{y y}-\Omega_{x y}{ }^{2}$, 
$A_{0}=\left[(Q+4)^{2}(Q-4)-4 Q R\right] \lambda_{0}{ }^{2}-R(Q+4)-4 R^{2}$,

$A_{1}=-8 \lambda_{0} R\left[2 \lambda_{0}^{2}-(Q+4)\right]$,

$A_{2}=-\lambda_{0}{ }^{2} R\left[Q^{2}-4 R-16\right]$,

$N=\left[4 Q^{2}+8 Q+4-16 R\right] \lambda_{0}{ }^{2}-4 R+(Q+1)^{2}$,

$F_{0}=\frac{1}{N}\left[\lambda_{0}^{2}(Q+1)+(Q+1)+2 R\right]$,

$F_{1}=\frac{-\lambda_{0}}{N}\left[2 \lambda_{0}^{2}+(Q+3)\right]$,

$F_{2}=\frac{-1}{N}\left[6 \lambda_{0}{ }^{2}-(Q+1)\right]$.

For transition curves of periodic solutions, the exponent will be

$\lambda=i\left(n \pm \frac{i}{2}\right), n=0, \pm 1, \pm 2$.

To get the curve in the range $0 \leq \mu \leq 1 / 2, n$ must be equal to 0 . Thus, the exponent should be

$\lambda= \pm \frac{i}{2}$

Equating Eq. (21) and Eq. (22),

$\left(\lambda_{0}+\lambda_{2} e^{2}\right)^{2}=-\frac{1}{4}$

Substituting $\lambda_{0}$ and $\lambda_{2}$ of this particular problem in Eq. (23) the transition curves (Fig 2) can be obtained. The region inside these curves represent the stable region in the plane. The transition curves shrink a little (minute variation) because of the perturbations.

i) $\quad A_{1}=0, \epsilon=0$

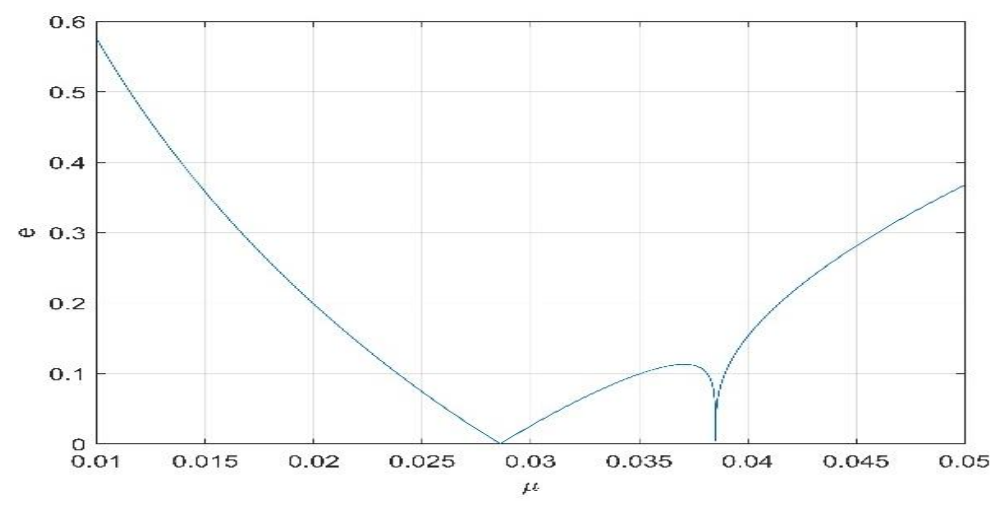

ii) $\quad A_{1}=0.0006, \epsilon=0$

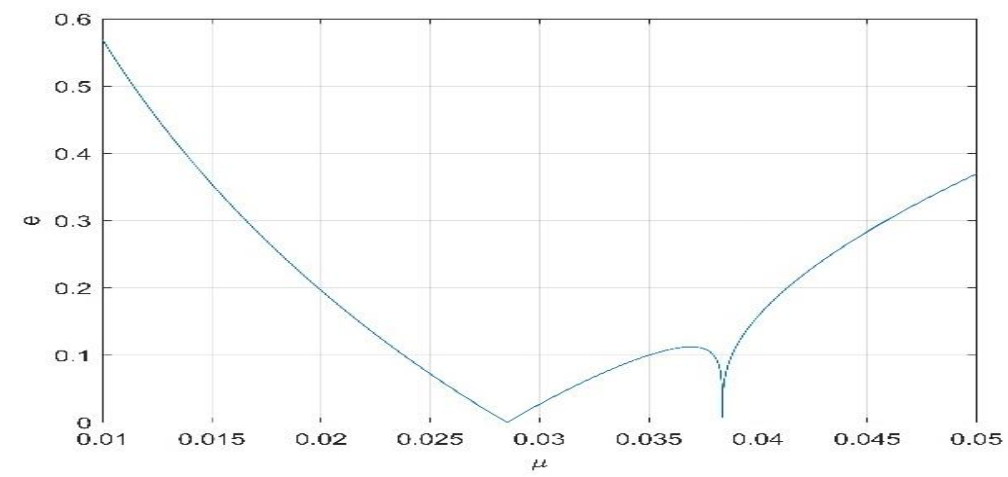

iii) $\quad A_{1}=0, \epsilon=0.0001$ 


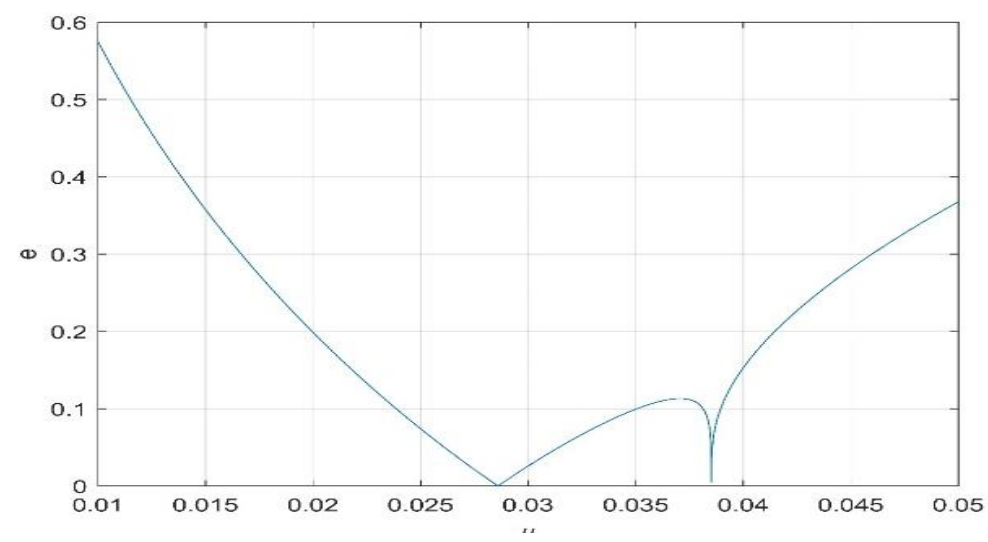

iv) $A_{1}=0.0006, \epsilon=0.0001$

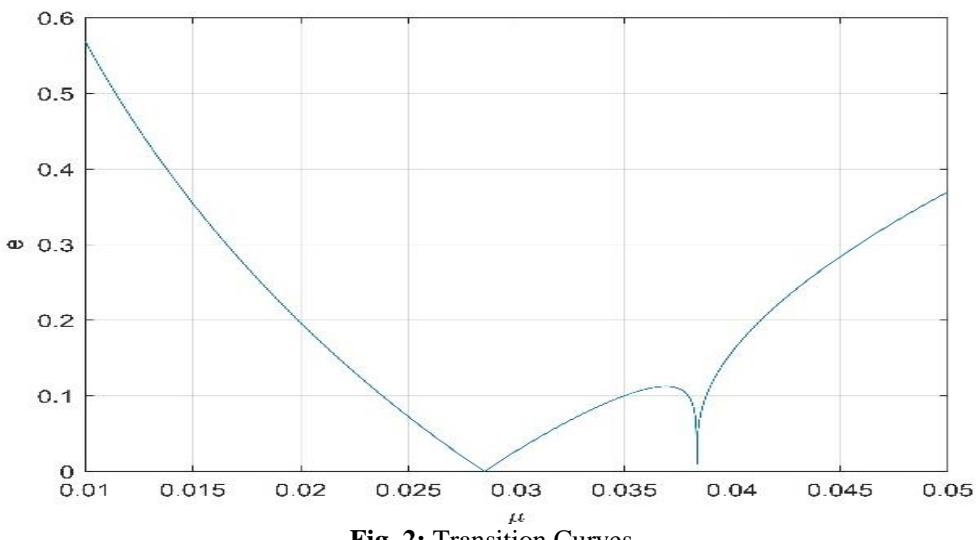

Fig. 2: Transition Curves.

\section{Conclusion}

The elliptic restricted three-body problem is studied for the case where the more massive primary is an oblate radiating spheroid. The equations of motion are formulated. The mean motion equation is obtained by analytical integration from the secular perturbation terms due to oblateness. The triangular Lagrangian points are located and their stability is analysed. The points move towards the primaries in the $\boldsymbol{y}$-direction. The limiting eccentricity for the stable system does not vary. The critical mass ratio reduces when compared to the earlier studies for the same case. Thus, the effect of the modified mean motion is felt only in the location and the critical mass ratio and not in the limiting eccentricity.

\section{References}

[1] L. Bucci, M. Lavagna, D. Guzetti, K. C. Howell, Periodic orbit-attitude solutions along planar orbits in a perturbed circular restricted three-body problem for the Earth-Moon system, Acta Astronautica Vol. 147 (2018) 152-162. https://doi.org/10.1016/j.actaastro.2018.03.042.

[2] Y. Meng, R. Hao, Q. Chen, Attitude stability analysis of a dual-spin spacecraft in halo orbits, Acta Astronautica, Vol. 99 (2014) 318-329. https://doi.org/10.1016/j.actaastro.2014.03.001.

[3] V. Szebehely, Theory of Orbits: The Restricted Problem of Three Bodies. Academic Press, New York, (1967). https://doi.org/10.1016/B978-0-12395732-0.50007-6.

[4] P. V. Subbarao, R. K. Sharma, A Note on the Stability of the Triangular Points of Equilibrium in the Restricted Three-body Problem, Astronomy and Astrophysics, Vol. 43 (1975) 381-383.

[5] J.M.A. Danby, Stability of the Triangular Points in the Elliptic Restricted Problem of Three Bodies, The Astronomical Journal, Vol. 69 No. 2 (1964) 165-172. https://doi.org/10.1086/109254.

[6] E. A. Grebenikov, On the Stability of the Lagrangian Triangle Solutions of the Restricted Elliptic Three-Body Problem, Soviet Astronomy - AJ, Vol. 8 No.3 (1964) 451-459.

[7] R. K. Sharma, on linear stability of triangular libration points of the photo-gravitational three- body problem when the more massive primary is an oblate spheroid, Sun and Planetary System, W. Fricke and G. Teleki (Eds.), D. Reidel Publishing Co., Dordrecht, Holland, (1982) $435-436$. https://doi.org/10.1007/978-94-009-7846-1_114.

[8] A. Narayan, C. R. Kumar, Stability of Triangular Equilibrium Points in Elliptical Restricted Three Body Problem under the effects of Photo-gravitational and Oblateness of primaries, International Journal of Pure and Applied Mathematics, Vol. 70 No. 5 (2011) 735-754.

[9] J. Singh, U. Aishetu, Motion in the Photo-gravitational Elliptic Restricted Three-body Problem under an Oblate Primary, The Astronomical Journal, Vol. 143 No. 5 (2012) 109-130. https://doi.org/10.1088/0004-6256/143/5/109.

[10] Y. S. Ruth, R. K. Sharma, Periodic Orbits in the Photo-gravitational Elliptic Restricted Three-Body Problem, Advances in Astrophysics, Vol. 3 No. 3 (2018) 154-170.

[11] R.K. Sharma, H. Sellamuthu, H. Isravel, Effect of oblateness on the locations and linear stability of collinear points in elliptic restricted three-body problem, communicated to Planetary and Space Science Journal (August 2018)

[12] H. D. Curtis, Orbital Mechanics for Engineering Students, 3rd Ed. Elsevier Aerospace Engineering Series, Elsevier, Amsterdam, (2014). https://doi.org/10.1016/B978-0-08-097747-8.00006-2.

[13] A. Bennett, Analytical Determination of Characteristic Exponents, AIAA/ION Astrodynamics Specialist Conference, California, (1965). https://doi.org/10.2514/6.1965-685. https://doi.org/10.2514/6.1965-685. 